\title{
Caractérisation phénotypique des plantules de quatre populations de Sclerocarya birrea (A. Rich.) Hochst. au Burkina Faso
}

Fatimata Anna DiALlo ${ }^{1}$ Abdoulazize SANDWIDI ${ }^{1}$ Madjelia Ebou Cangré DAO ${ }^{1}$ Pauline BATIONO-KANDO ${ }^{2}$ Boukary Ousmane DIALLO ${ }^{1}$

${ }^{1}$ Institut de l'environnement et de recherches agricoles Département Environnement et Forêts BP 7047, Ouagadougou 03 Burkina Faso

2 Université Joseph Ki-Zerbo Unité de formation et de recherche en sciences de la Vie et de la Terre Laboratoire Bioscience 09 BP 7021, Ouagadougou 03 Burkina Faso

\section{Auteur correspondant /} Corresponding author: Fatimata Anna DIALLO annadiallofat@gmail.com

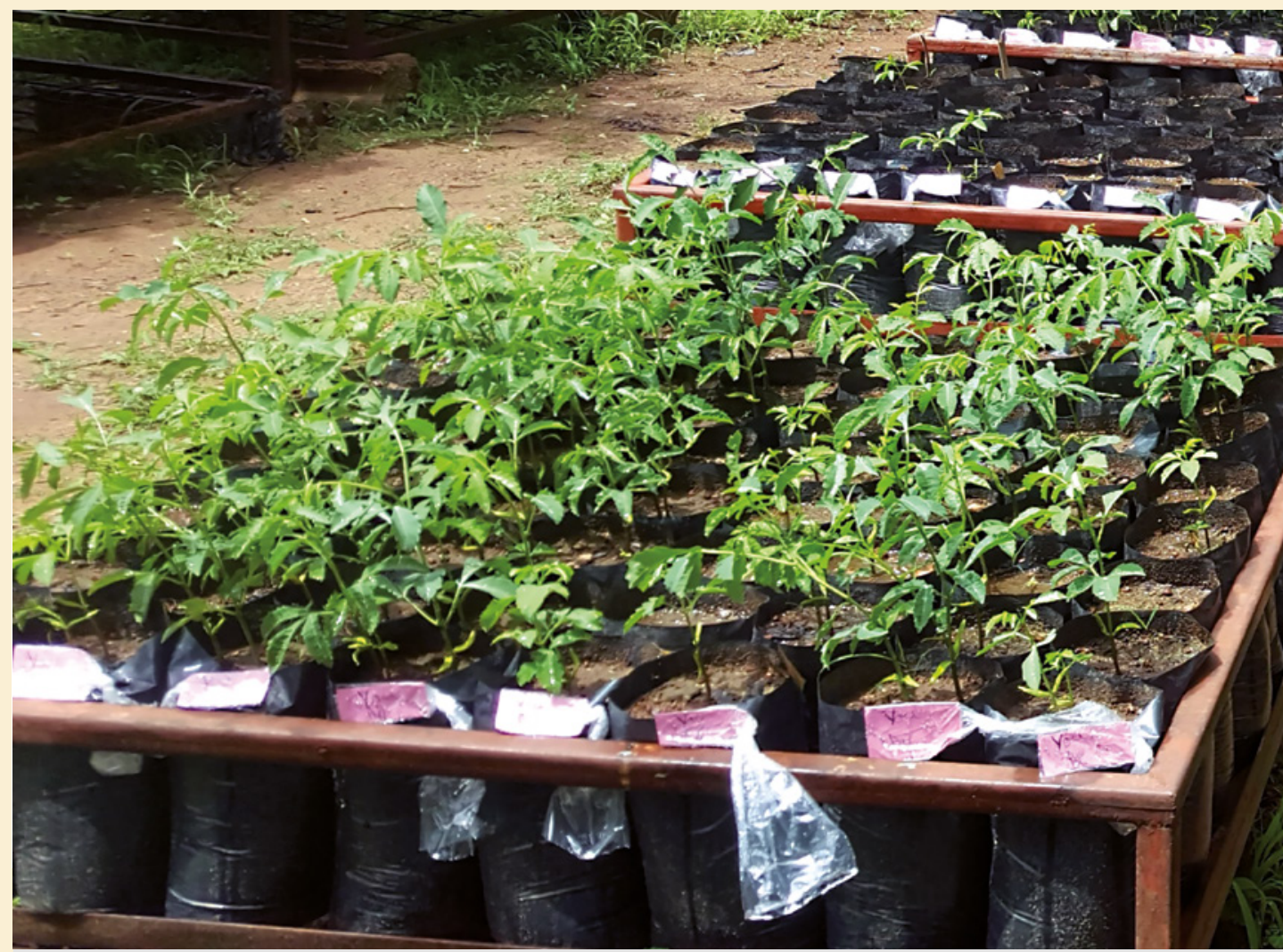

Photo 1.

Plantules de Sclerocarya birrea utilisées pour l'essai. Photo Diallo et al., 2020.

Doi : 10.19182/bft2020.344.a31898 - Droit d'auteur (c) 2020, Bois et Forêts des Tropiques @ Cirad - Date de soumission : 23 septembre 2019 ; date d'acceptation : 13 avril 2020 ; date de publication : $1^{\text {er juin }} 2020$.

\section{Ocirad (6)}

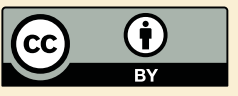

Citer l'article / To cite the article

Diallo F. A., Sandwidi A., Dao M. E. C., Bationo-Kando P., Diallo B. O., 2020. Caractérisation phénotypique des plantules de quatre populations de Sclerocarya birrea (A. Rich.) Hochst. au Burkina Faso. Bois et Forêts des Tropiques, 344 : 33-46. Doi: https://doi.org/10.19182/bft2020.344.a31898 


\section{RÉSUMÉ}

\author{
Caractérisation phénotypique \\ des plantules de quatre populations \\ de Sclerocarya birrea (A. Rich.) Hochst. \\ au Burkina Faso
}

Sclerocarya birrea (A. Rich.) Hochst. est un fruitier oléagineux d'Afrique. Les fortes mortalités après plantation sont dues à l'affectation inappropriée des plants dans les zones écologiques. La caractérisation phénotypique des plantules a pour objectif de déterminer dès le jeune âge des caractères d'adaptation aux conditions écologiques. L'étude porte sur 494 plantules originaires des populations de You, Yarci, Gonsé et Diapangou-Peulh au Burkina Faso. Celles-ci appartiennent à quatre localités circonscrites dans trois zones climatiques. Les résultats montrent que la levée groupée assure la présence de générations chevauchantes à l'intérieur des populations. L'analyse de variance qui fait apparaître des différences intra- et interpopulations sur la majorité des variables traduit la diversité phénotypique des plantules. L'analyse en composantes principales montre des caractéristiques spécifiques à chaque population. Le dendrogramme de la classification ascendante hiérarchisée permet d’identifier trois groupes : G1 : Gonsé (55,88\%), Yarci $(23,52 \%)$ et You $(20,58 \%)$; G2 : You (46,15\%), Yarci (42,30\%), Diapangou-Peulh $(7,69 \%)$ et Gonsé (3,84\%) ; G3 : Diapangou-Peulh (90\%), Yarci (5\%) et You (5\%). Ces trois groupes traduisent le rapprochement phénotypique entre individus. Ces résultats mettent en évidence l'existence des gains génétiques pour la sélection individuelle et populationnelle. Ils montrent également que ces populations ne sont pas menacées d'impasse évolutive à court terme. L'étude des héritabilités indique que les caractères les plus héritables sont la hauteur de la tige $(28,31 \%)$, la longueur de la racine principale (44,30\%), le nombre de racines secondaires $(81,89 \%)$, le poids frais des racines $(73,47 \%)$ et le poids sec des racines $(28,86 \%)$. L'architecture du système racinaire traduit la capacité d'adaptation de Sclerocarya birrea à différents types de sols. Compte tenu de ces résultats, la sélection sur les caractères adaptatifs au jeune âge peut être envisagée.

Mots-clés : Sclerocarya birrea, caractères phénotypiques, adaptation, stratégie de levée, héritabilité, sélection, Burkina Faso.

\section{ABSTRACT}

\section{Phenotype characterisation of seedlings from four Sclerocarya birrea (A. Rich.) Hochst. populations in Burkina Faso}

Sclerocarya birrea (A. Rich.) Hochst. is an African oleaginous fruit tree. High post-plantation mortality is due to planting in inappropriate ecological zones. This study characterised the seedling phenotypes in order to determine their ecologically adapted traits from a very early stage. A total of 494 seedlings were analysed, from populations in You, Yarci, Gonsé and Diapangou-Peulh in Burkina Faso, four locations that fall within three different climatic zones. The study results show that grouped seedling emergence ensures that overlapping generations are present within each population. Variance analysis showed differences within and between populations for most variables, reflecting the diversity of phenotypes among the seedlings. Principal Component Analysis showed characteristics specific to each population. The dendrogramme for ranked classification in ascending order identified three groups of individual seedlings with similar phenotypes: G1 for Gonsé (55.88\%), Yarci (23.52\%) and You (20.58\%); G2 for You (46.15\%), Yarci (42.30\%), Diapangou-Peulh (7.69\%) and Gonsé (3.84\%); G3 for Diapangou-Peulh (90\%), Yarci (5\%) and You (5\%). These results show the existence of genetic gains in selection at the individual and population level. They also show that these populations are not in danger of coming to an evolutionary dead-end in the short term. The study of heritability showed that the most heritable traits are stem height (28.31\%), length of the primary root (44.30\%), number of secondary roots (81.89\%), fresh weight of the roots $(73.47 \%)$ and dry weight of the roots (28.86\%). The root system architecture reflects the adaptation capacity of Sclerocarya birrea to different soil types. In view of these results, selective breeding for adaptive traits may be considered at an early stage.

Keywords: Sclerocarya birrea, phenotypic traits, adaptation, emergence strategy, heritability, selection, Burkina Faso.

\section{RESUMEN}

\author{
Caracterización fenotípica \\ de las plántulas de cuatro poblaciones \\ de Sclerocarya birrea (A. Rich.) Hochst. \\ en Burkina Faso
}

La Sclerocarya birrea (A. Rich.) Hochst. es un frutal oleaginoso de África. La importante mortalidad después de la plantación es debida a la selección de zonas ecológicas inadecuadas. La caracterización fenotípica de las plántulas tiene como objetivo determinar desde una edad temprana los caracteres de adaptación a las condiciones ecológicas. El estudio se realizó en 494 plántulas originarias de las poblaciones de You, Yarci, Gonsé y Diapangou-Peulh, en Burkina Faso. Estas cuatro localidades se encuentran en tres zonas climáticas diferentes. Los resultados muestran que la emergencia agrupada de brotes garantiza la presencia de generaciones solapadas en el interior de las poblaciones. El análisis de varianza, que manifiesta diferencias intrapoblacionales e interpoblacionales en la mayor parte de las variables, expresa la diversidad fenotípica de las plántulas. El análisis de componentes principales muestra características específicas de cada población. El dendrograma de la clasificación ascendente jerarquizada permite identificar tres grupos: G1: Gonsé (55,88\%), Yarci $(23,52 \%)$ y You (20,58 \%); G2: You (46,15\%), Yarci (42,30\%), Diapangou-Peulh (7,69\%) y Gonsé (3,84\%), y G3: Diapangou-Peulh (90\%), Yarci (5\%) y You (5\%). Los tres grupos manifiestan la proximidad fenotípica entre individuos. Estos resultados evidencian que hay ganancias genéticas para la selección individual y poblacional. Muestran asimismo que estas poblaciones no están amenazadas con un punto muerto evolutivo a corto plazo. El estudio de las heredabilidades indica que: los caracteres más heredables son la altura del tallo $(28,31 \%)$, la longitud de la raíz principal (44,30\%), el número de raíces secundarias $(81,89 \%)$, el peso húmedo de las raíces $(73,47 \%)$ y el peso seco de las raíces (28,86\%). La arquitectura del sistema radical revela la capacidad de adaptación de Sclerocarya birrea a diferentes tipos de suelo. Teniendo en cuenta estos resultados, se puede plantear la selección de los caracteres adaptativos en edad temprana.

Palabras clave: Sclerocarya birrea, caracteres fenotípicos, adaptación, estrategia de emergencia, heredabilidad, selección, Burkina Faso. 


\section{Introduction}

$\mathrm{Au}$ Sahel, les produits forestiers non ligneux font l'objet d'un important commerce sur les marchés locaux (Lamien et Bayala, 1996). Ils constituent une source de revenus pour les populations rurales (Avana et al., 2019). Parmi ces produits, les fruits des espèces locales ont été intégrés comme un nouveau levier de développement dans les programmes nationaux du secteur rural (PNSR). C'est le cas des fruits de Sclerocarya birrea (A. Rich.) Hochst., communément appelé prunier d'Afrique. Cette espèce, objet de la présente étude, est un fruitier oléagineux endémique à l'Afrique et appartenant à la famille des Anacardiaceae (Hall, 2002). La pulpe de ses fruits est utilisée pour la fabrication de jus et de boisson alcoolisée. Cette boisson alcoolisée est commercialisée sous le nom de «kouna doro» en Afrique de l'Ouest. L'amande de ses fruits est utilisée pour l'extraction d'huile destinée à l'exportation, principalement pour les industries pharmaceutiques (Faye et al., 2011). Au Burkina Faso, plusieurs petites unités de transformation détenues par des associations et groupements de femmes (Union Yanta, Femme 2000, Union des femmes de Kossougdou) se sont spécialisées dans la fabrication de jus et/ou l'extraction d'huile dont la valeur marchande est d'environ 12000 francs CFA le litre sur les marchés locaux (Faye et al., 2011). Du fait de l'intérêt que suscitent les fruits de Sclerocarya birrea, les populations de cette espèce sont soumises à de fortes exploitations. Or, en l'absence de plantations de production et de plans de gestion appropriés des forêts primaires et des parcs agroforestiers, les productions ne couvrent plus les besoins de ces petites unités de transformation. C'est dans ce contexte que le ministère en charge de l'environnement a entrepris depuis une décennie des actions de reboisement participatif, sous forme d'enrichissement des parcs agroforestiers et des peuplements spontanés. Cependant, des rapports techniques du ministère ont fait état de taux de mortalité très élevés, compris entre 60 et $90 \%$ suivant les sites de plantation (Direction générale des forêts, 2016). Toutefois, aucune cause n'a été avancée pour expliquer de tels résultats. Diallo et al. (2012) mentionnent qu'en zone soudanienne et sahélienne les fortes mortalités observées après reboisement sont principalement dues à une affectation inappropriée du matériel végétal dans les zones de plantation. Partant de cette observation et pour pallier les fortes mortalités, il paraît nécessaire de sélectionner le matériel végétal avant plantation sur la base des caractères adaptatifs. Ceci passe nécessairement par des tests d'adaptation dont les plus courants sont les essais de provenance en milieu réel (Billand et Diallo, 1991). Cependant, la mise en place de tels dispositifs, ainsi que leur maintien à long terme, sont coûteux pour les pays en développement (Diallo et al., 2000 ; Weber et al., 2018). De plus, l'indicateur utilisé pour évaluer le niveau d'adaptation dans les essais de provenance est le taux de survie. Il s'agit d'une évaluation à postériori qui rend l'âge de sélection tardif et aléatoire. De ce fait, pour raccourcir les schémas de sélection et minimaliser les coûts engendrés par les essais de provenance en milieu réel, de nouvelles approches d'évaluation du matériel végétal à sélectionner doivent être envisagées. À ce sujet, Diallo et al. (2010) et Weber et al. (2018) ont montré que les caractéristiques des plantules, dès l'âge de la pépinière, sont des indicateurs pour la sélection fondée sur l'adaptation du matériel végétal aux conditions écologiques. En effet, les informations recueillies à ce stade où les effets maternels sont très forts sont fondamentales pour établir des corrélations jeunes/ adultes. Ceci permet d'opérer une sélection précoce sur le critère d'adaptation (Diallo, 2017).

Hall (2005) indique que les populations du prunier d'Afrique auraient probablement pour origine la dissémination des graines par les chauves-souris, les ruminants et les primates (singe, homme) dans différentes zones écologiques. Cependant, Diallo et al. (2010) ont montré que de telles populations ont pu acquérir des caractères adaptatifs spécifiques en réponse à leur environnement. Ces processus sont liés à l'effet de la sélection naturelle due aux forces évolutives locales.

De plus, l'analyse de la diversité génétique des populations conduite par Gutman et al. (1999), Muok etal. (2007, 2011) et Kando et al. (2012) met en évidence une forte différenciation génétique chez le prunier d'Afrique. De ce fait, on peut penser que cette différenciation génétique a pu induire une différenciation phénotypique pour des populations ayant évolué dans des conditions écologiques variées, comme l'avaient mentionné Hébert et Vincourt (1985). Cette relation génotype/phénotype a été confirmée par les travaux de Shackleton (2002) et ceux de Kando et al. (2008) sur les caractères morpho-adaptatifs des arbres adultes de différentes populations.

Toutefois, malgré l'intérêt scientifique de ces travaux, les données au jeune âge sont quasi inexistantes. Pourtant, les informations recueillies à ce stade sont de première importance dans les schémas de sélection des ligneux (Diallo et al., 2000).

L'objectif de cette étude est de déterminer les potentialités d'adaptation de plantules de Sclerocarya birrea à leur environnement, à partir de leurs stratégies de levée et de leurs caractéristiques phénotypiques. L'hypothèse générale est que la stratégie de levée et les caractéristiques phénotypiques des plantules seraient des caractéristiques spécifiques liées à l'origine écologique du matériel végétal. Cinq sous-hypothèses sont alors émises : le type de levée serait fonction de l'aire d'origine des semences ; les caractéristiques des plantules permettraient de différencier le matériel végétal en fonction de l'aire d'origine des semences ; au jeune âge, l'héritabilité varierait en fonction des types de caractères adaptatifs ; il existerait des descripteurs phénotypiques des plantules qui sont spécifiques à chaque population; le compromis d'allocation des ressources entre la partie aérienne et la partie souterraine dès le jeune âge dépendrait de l'aire d'origine des semences. 


\section{Matériels et méthodes}

\section{Sites de collecte des semences}

Le matériel végétal a été récolté dans quatre localités du Burkina Faso suivant un gradient climatique Nord/Sud (figure 1). Selon Fontès et Guinko (1995), ces quatre localités se caractérisent par une végétation variée avec des degrés de fermeture croissants allant du Nord au Sud. II s’agit des localités de :

- You, située dans la province du Loroum dans le département de Titao, en zone sahélienne, et caractérisée par une végétation de steppe arborée ;

- Yarci, située dans la province du Passoré dans le département de Arbolé, en zone nord-soudanienne, et caractérisée par une végétation de brousse tigrée ;

- Gonsé, située dans la province du Kadiogo dans le département de Saaba, en zone nord-soudanienne, et caractérisée par une végétation de savane arborée et arbustive ;

- Diapangou-Peulh, située dans la province du Gourma dans le département de Diabo, dans une zone de transition entre la zone nord-soudanienne et la zone soudanienne elle est caractérisée par une végétation de savane arborée et arbustive avec une grande diversité d'espèces.

\section{Site d'expérimentation}

Les travaux ont été menés dans l'une des pépinières expérimentales du département Environnement et Forêts (DEF), à l'intérieur du parc botanique du Centre national de la recherche scientifique et technologique (CNRST) dont les coordonnées géographiques sont N $12^{\circ} 22.801^{\prime}$ (latitude Nord) et 0 01³0.295' (longitude Ouest). Ce parc, situé en climat nord-soudanien, se caractérise par un microclimat particulier induit par une végétation abondante qui est une relique de la forêt-galerie du barrage de Ouagadougou.

\section{Matériel végétal}

Le matériel végétal est constitué de plantules de Sclerocarya birrea (photo 1) obtenues à partir des graines récoltées sur des arbres-mères des quatre populations. Chaque population est circonscrite à l'une des quatre localités de récolte (You, Yarci, Gonsé et Diapangou-Peulh). La figure 2 illustre la cartographie des arbresmères de ces quatre populations qui ont été géoréférencées à l'aide d'un GPS. Le logiciel utilisé est ARC GIS 6.2.

Le tableau I donne les caractéristiques géo-pédoclimatiques de ces quatre localités.

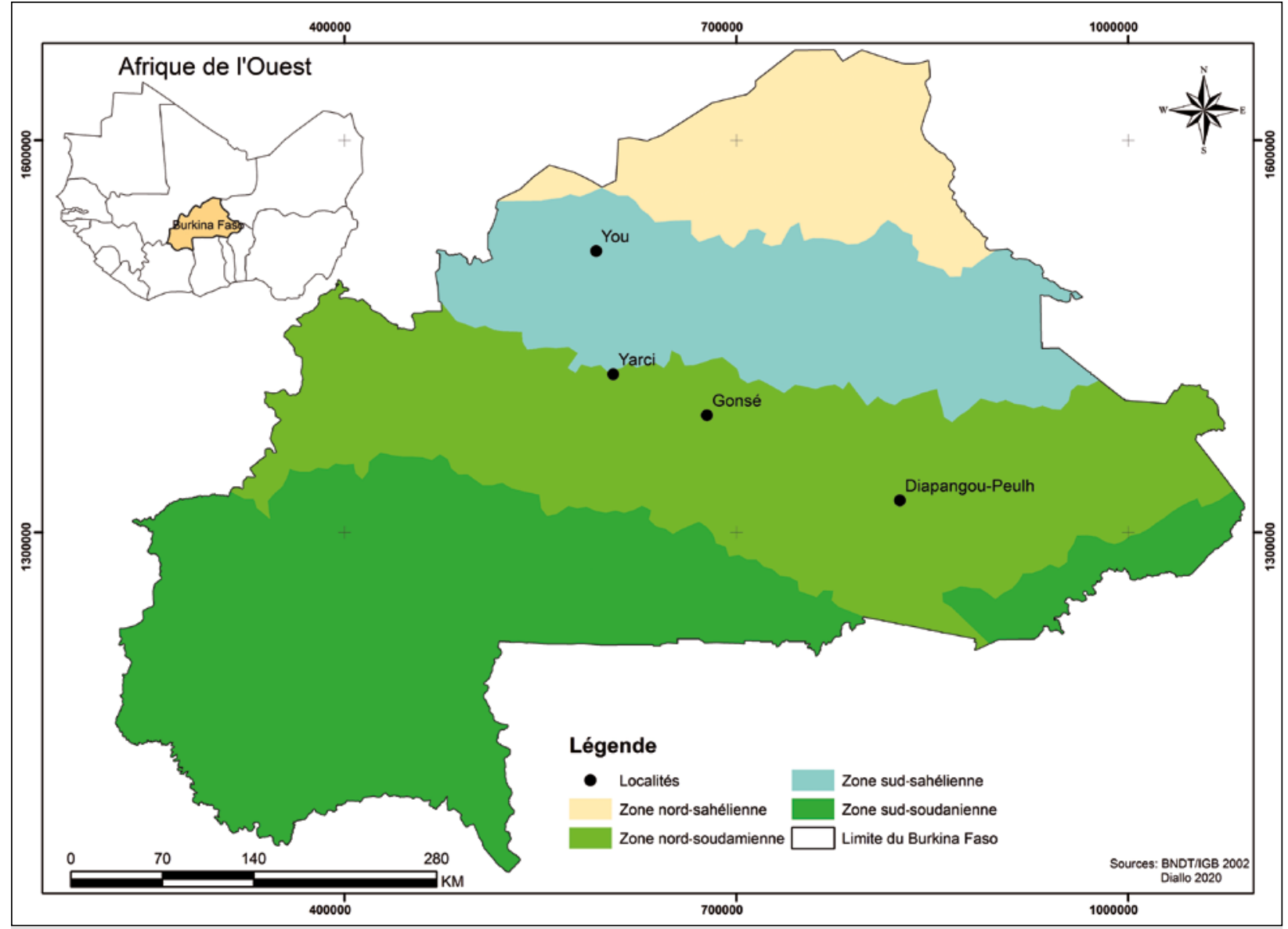

Figure 1.

Localisation des sites de collecte des graines de Sclerocarya birrea. 
Tableau I.

Caractéristiques géo-pédoclimatiques des sites de collecte des semences.

\begin{tabular}{|c|c|c|c|c|}
\hline Localité & Climat & $\begin{array}{c}\text { Relief } \\
\text { (topographie) }\end{array}$ & $\begin{array}{c}\text { Sols } \\
\text { (classification } \\
\text { française) }\end{array}$ & $\begin{array}{c}\text { Température } \\
\text { moyenne } \\
\text { annuelle }\end{array}$ \\
\hline You & $\begin{array}{l}\text { Climat sud-sahélien } \\
\text { Pluviosité annuelle : } \\
600 \text { mm }\end{array}$ & $\begin{array}{l}\text { Terrain plat avec deux } \\
\text { toposéquences (moyen } \\
\text { et bas glacis) }\end{array}$ & $\begin{array}{l}\text { Sols bruns } \\
\text { ferrugineux } \\
\text { tropicaux }\end{array}$ & $29^{\circ} \mathrm{C}$ \\
\hline Yarci & $\begin{array}{l}\text { Climat nord- } \\
\text { soudanien } \\
\text { Pluviosité annuelle : } \\
700 \mathrm{~mm}\end{array}$ & $\begin{array}{l}\text { Terrain plat avec deux } \\
\text { toposéquences (moyen } \\
\text { et bas glacis) }\end{array}$ & $\begin{array}{l}\text { Sols tropicaux } \\
\text { ferrugineux lessivés } \\
\text { à tache et concrétion }\end{array}$ & $28^{\circ} \mathrm{C}$ \\
\hline Gonsé & $\begin{array}{l}\text { Climat nord- } \\
\text { soudanien/soudanien } \\
\text { pluviosité annuelle : } \\
800 \mathrm{~mm}\end{array}$ & $\begin{array}{l}\text { Terrain plat avec deux } \\
\text { toposéquences (moyen } \\
\text { et bas glacis) }\end{array}$ & $\begin{array}{l}\text { Sols ferrugineux } \\
\text { tropicaux argilo-sableux } \\
\text { localement sur dalle } \\
\text { latéritique indurée }\end{array}$ & $28^{\circ} \mathrm{C}$ \\
\hline Diapangou-Peulh & $\begin{array}{l}\text { Climat soudanien } \\
\text { Pluviosité annuelle : } \\
800 \text { à } 900 \mathrm{~mm}\end{array}$ & $\begin{array}{l}\text { Terrain plat avec une } \\
\text { toposéquence (haut } \\
\text { glacis) }\end{array}$ & $\begin{array}{l}\text { Sols ferrugineux } \\
\text { tropicaux lessivés indurés, } \\
\text { moyennement profonds }\end{array}$ & $27^{\circ} \mathrm{C}$ \\
\hline
\end{tabular}

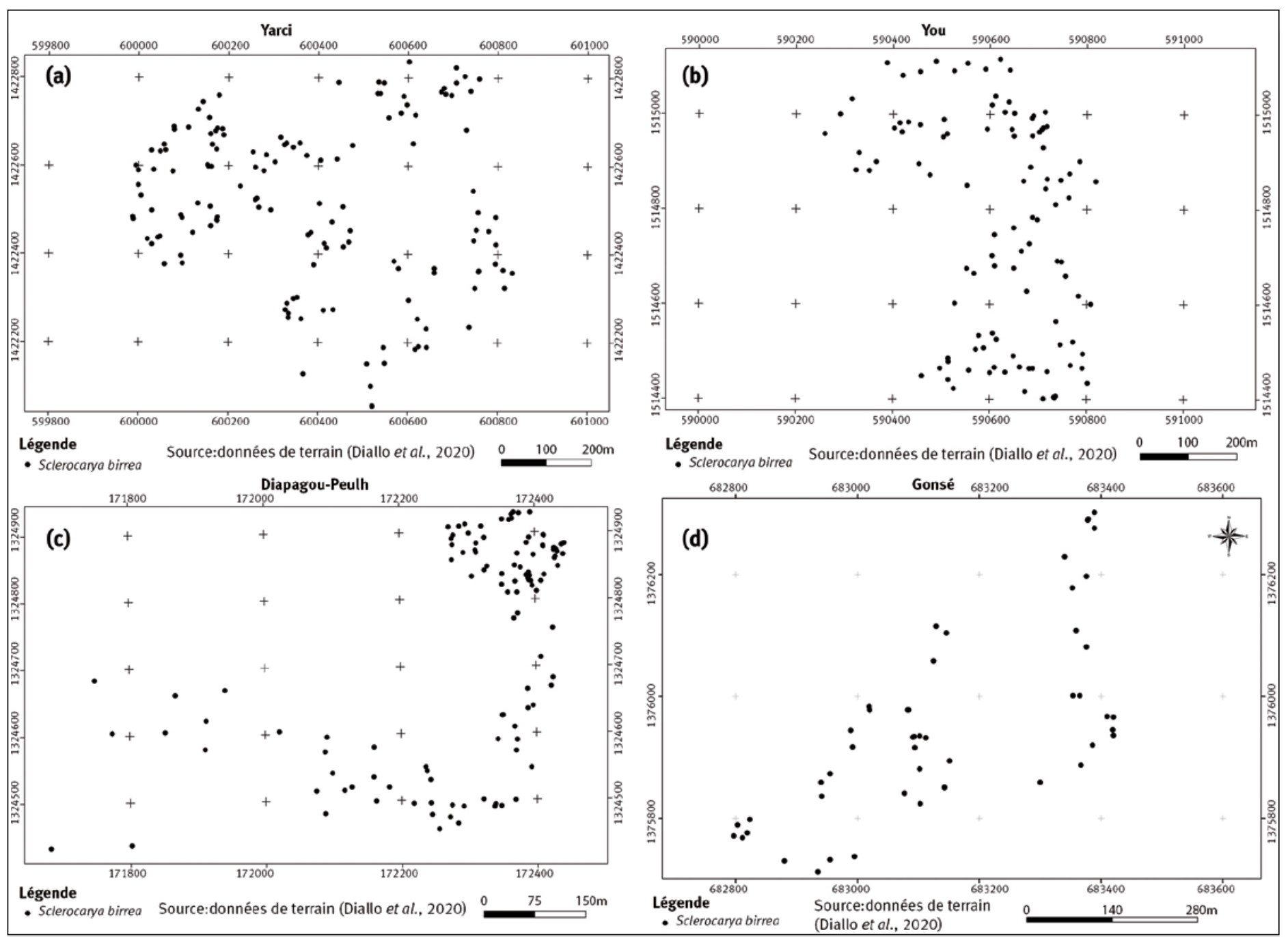

Figure 2.

Cartographie des arbres-mères échantillonnés pour les quatre populations. 


\section{Récolte des fruits}

Les récoltes ont été faites suivant les normes internationales définies à la conférence de Stockholm (Garnier, 1972). Celles-ci sont établies sur la distance entre les arbres-mères, leur état sanitaire et la taille de l'échantillon. Compte tenu du caractère androdioïque de l'espèce (Diallo et al., 2006), 20 arbres visuellement sains, distants l'un de l'autre d'environ 100 m, ont été échantil-

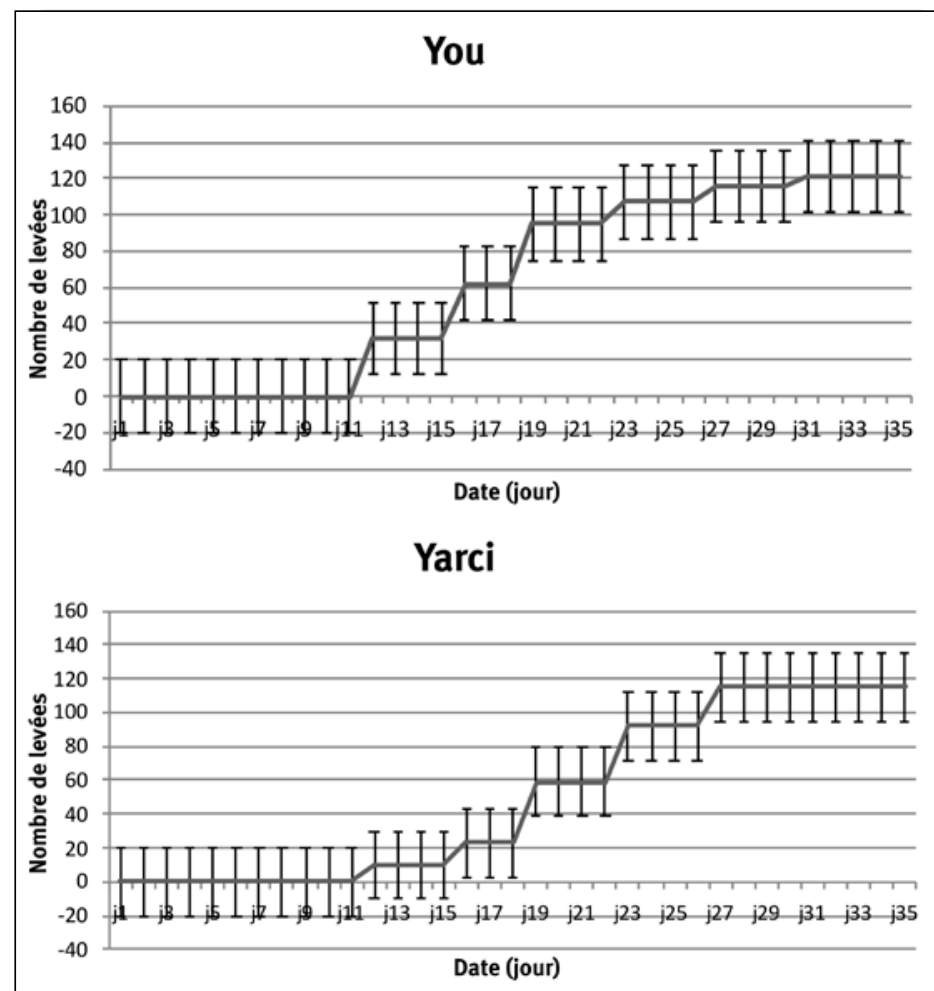

Gonsé

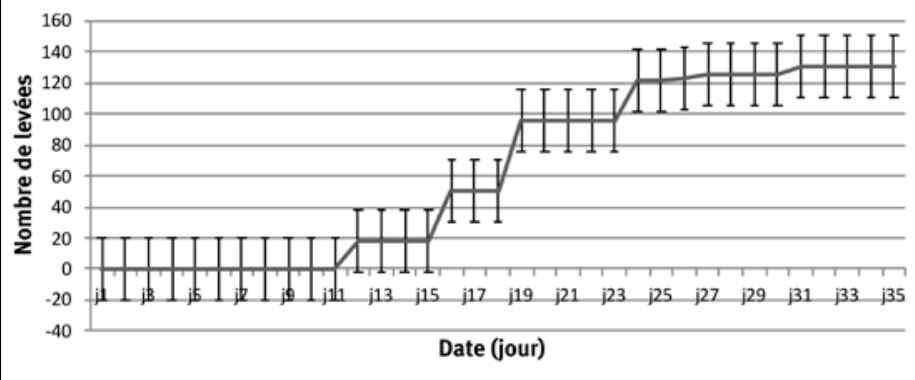

Diapangou-Peulh

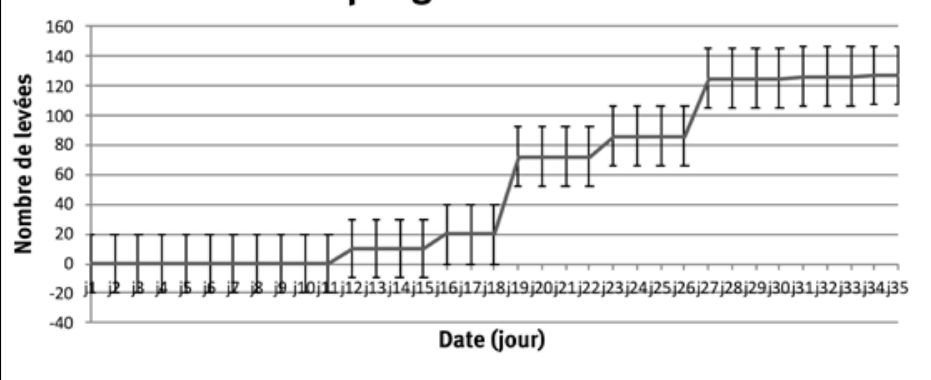

Figure 3.

Courbes de levée des plantules pour les quatre populations. lonnés au sein de chaque population ; 40 fruits arrivés à maturité commerciale ont été récoltés sur chaque arbre.

\section{Mise en place des semis et suivi de germination}

Les fruits ont été épluchés dans l'eau ; 8 graines issues des 40 fruits ont été choisies au hasard puis semées sans prétraitement particulier, dans des conteneurs en polyéthylène de dimension $30 \mathrm{~cm} \times 10 \mathrm{~cm}$. Ceux-ci contiennent un mélange de deux tiers de terre et un tiers de sable. Les graines ont été semées le 7 juin 2018 à raison d'une graine par conteneur. Afin d'éviter les attaques des rongeurs, les conteneurs ont été montés sur des châssis disposés de façon aléatoire. Chaque population est représentée par deux châssis contenant 10 lignes de 8 graines, chaque ligne correspondant à un arbremère. On a ainsi un total de 20 lignes de graines, soit 160 graines semées par population. Après les semis, la germination a été suivie de deux façons : durant les deux premières semaines, suivi quotidien afin de connaître la date de la première germination; après deux semaines et pendant 35 jours, suivi bihebdomadaire pour déterminer le type de levée. Le comptage correspond au cumul du nombre de levées à chaque passage.

\section{Variables étudiées}

Pour la caractérisation morphologique des plantules, après 4 mois de semis ( 5 octobre 2018), 5 plantules par arbre-mère ont été échantillonnées, soit 100 plants par population, d'où un total de 400 plants parmi les 494 plants germés. Les parties aériennes ont été séparées des parties souterraines à l'aide d'un sécateur puis conditionnées dans des enveloppes étiquetées. Les tiges, les feuilles et les racines ont été pesées séparément à l'état frais puis séchées dans une étuve électrique à $60^{\circ} \mathrm{C}$ pendant $72 \mathrm{~h}$.

Dix variables ont été mesurées, quatre variables ont été calculées et des comptages ont été effectués sur deux variables.

Le diamètre au collet (Dc) et le diamètre de la racine principale (Drp) ont été mesurés à l'aide d'un pied à coulisse digital. La hauteur de la tige $(\mathrm{Ht})$ et la longueur de la racine (Lrp) ont été mesurées à l'aide d'un mètre ruban. Le poids frais de la tige (Pft), le poids sec de la tige (Pst), le poids frais des feuilles (Pff), le poids sec des feuilles (Psf), le poids frais des racines (Pfr) et le poids sec des racines (Psr) ont été déterminés à l'aide d'une balance électrique. Le nombre de rameaux primaires (Nram) et le nombre de racines secondaires (Nrs) ont été comptés de façon exhaustive. Les quatre variables calculées portent sur les héritabilités au sens large des différentes variables et les rapports entre variables.

\section{Traitements statistiques des données}

Le tableur Excel 2010 a été utilisé pour la saisie des données et la réalisation des courbes de levée. Les analyses statistiques des données ont été effectuées avec 
Tableau II.

Résultats de l'analyse de variance intra-population.

\begin{tabular}{|c|c|c|c|c|c|c|c|c|}
\hline \multirow[b]{2}{*}{ Variables } & \multicolumn{2}{|c|}{ You } & \multicolumn{2}{|c|}{ Yarci } & \multicolumn{2}{|c|}{ Gonsé } & \multicolumn{2}{|c|}{ Diapangou-Peulh } \\
\hline & $\mathbf{F}$ & $\mathbf{P}$ & $\mathbf{F}$ & $\mathbf{P}$ & $\mathbf{F}$ & $\mathbf{P}$ & $\mathbf{F}$ & $\mathbf{P}$ \\
\hline Ht & 1,55 & $0,009^{* *}$ & 1,07 & $0,39 \mathrm{~ns}$ & 1,89 & $0,03^{*}$ & 2,56 & $0,007^{\star \star}$ \\
\hline Dc & 1,45 & $0,13 n s$ & 1,02 & $0,44 \mathrm{~ns}$ & 1,10 & $0,36 \mathrm{~ns}$ & 1,05 & 0,40ns \\
\hline Nram & 1,01 & $0,46 n s$ & 1,12 & $0,35 \mathrm{~ns}$ & 1,03 & $0,43 n s$ & 1,02 & $0,47 \mathrm{~ns}$ \\
\hline Pft & 1,82 & $0,04^{\star}$ & 1,05 & $0,41 \mathrm{~ns}$ & 1,75 & $0,05^{\star}$ & 0,55 & $0,69 \mathrm{~ns}$ \\
\hline Pst & 2,71 & $0,002^{\star \star}$ & 1,14 & $0,33 n s$ & 1,14 & $0,32 \mathrm{~ns}$ & 0,65 & $0,62 \mathrm{~ns}$ \\
\hline Pff & 2,69 & $0,002^{\star \star}$ & 3,23 & $0,0001^{\star \star \star}$ & 2,24 & $0,008^{\star \star}$ & 0,31 & $0,86 \mathrm{~ns}$ \\
\hline Psf & 2,61 & $0,002^{\star \star}$ & 2,61 & $0,002^{\star \star}$ & 1,15 & $0,32 \mathrm{~ns}$ & 0,70 & $0,59 \mathrm{~ns}$ \\
\hline Lrp & 3,40 & $0,0001^{* * *}$ & 1,46 & $0,12 \mathrm{~ns}$ & 1,78 & $0,04^{*}$ & 4,31 & $0,01^{*}$ \\
\hline Drp & 3,31 & $0,0001^{\star \star \star}$ & 1,03 & $0,43 n s$ & 1,50 & $0,11 \mathrm{~ns}$ & 0,82 & $0,52 \mathrm{~ns}$ \\
\hline Nrs & 2,27 & $0,008^{\star \star}$ & 2,30 & $0,006^{\star \star}$ & 1,92 & $0,02^{*}$ & 1,64 & $0,20 \mathrm{~ns}$ \\
\hline Pfr & 3,15 & $0,0001^{\star \star \star}$ & 1,52 & $0,10 \mathrm{~ns}$ & 1,36 & $0,17 \mathrm{~ns}$ & 1,55 & $0,22 \mathrm{~ns}$ \\
\hline Psr & 5,32 & $0,0001^{\star * *}$ & 1,80 & $0,03^{*}$ & 1,66 & $0,006^{\star \star}$ & 1,62 & $0,20 \mathrm{~ns}$ \\
\hline
\end{tabular}

$\mathrm{P}$ : probabilité ; F : F statistique ; $\mathrm{Ht}(\mathrm{cm})$ : hauteur de la tige ; Dc $(\mathrm{mm})$ : diamètre au collet ; Nram : nombre de rameaux; Pft (g) : poids frais de la tige ; Pst (g) : poids sec de la tige ; Pff (g) : poids frais des feuilles ; Psf $(\mathrm{g})$ : poids sec des feuilles; $\operatorname{Lrp}(\mathrm{cm})$ : longueur de la racine principale; $\operatorname{Drp}(\mathrm{mm})$ : diamètre de la racine principale; Nrs : nombre de racines secondaires ; Pfr (g) : poids frais de la racine ; Psr (g) : poids sec de la racine ; ns : non significatif ; ${ }^{\star}$ : significatif ; ${ }^{\star \star}$ : très significatif ; ${ }^{\star \star \star}$ : hautement significatif.

le logiciel XLSTAT version 16.1. Pour les analyses statistiques, les deux facteurs non croisés «population " et « arbre » ont été étudiés. Pour chaque variable mesurée, une analyse de variance (ANOVA) suivie d'une comparaison de moyenne par le test de Newman-Keuls au seuil de $5 \%$ ont été réalisées. Celles-ci nous ont permis de différencier les arbres à l'intérieur de chaque population ainsi que les populations entre elles. Il faut noter qu'en matière de sélection un caractère n'est intéressant que s'il est transmis aux descendants avec une probabilité élevée. Ceci a conduit à calculer les héritabilités au sens large pour chaque variable suivant la formule appliquée par Nanson (2004) sur les populations des arbres forestiers: héritabilité au sens large $\left(\mathrm{H}^{2}\right)=$ (variance population $x$ 100) / (variance erreur - variance population).

Une analyse en composantes principales (ACP) réalisée à partir des données centrées réduites a permis d'établir la corrélation entre les individus et les variables, et de les positionner dans plusieurs plans bidimensionnels. Une classification ascendante hiérarchisée (CAH) dont le principe repose sur le calcul de distance euclidienne a permis de créer des groupes d'individus basés sur le principe du plus proche ressemblant phénotypique (Dagnelie, 1998). Le compromis d'investissement des ressources nutritives a été établi en calculant les rapports hauteur de la tige/longueur de la racine principale, poids frais aérien/poids frais souterrain et poids sec aérien/ poids sec souterrain.

\section{Résultats}

Type de levée

Les courbes de levée (figure 3) montrent que les premières levées ont été observées au $12^{\mathrm{e}}$ jour après semis pour les populations. La quasi-totalité des levées a été observée dès la quatrième semaine après semis, soit deux semaines après les premières levées.

\section{Caractéristiques phénotypiques des plantules}

\section{Variabilité des caractères}

Dans le tableau II, les résultats des analyses de variance effectuées sur chaque population prise individuellement montrent que certaines variables ne présentent pas de différences significatives entre individus. Cependant, aux seuils de probabilité $P$ de $5 \%, 1 \%$ et $0,1 \%$, on observe pour les autres variables des différences significatives à hautement significatives entre les individus.

À You, hormis Dc et Nram, toutes les autres variables présentent des différences significatives entre les individus.

À Yarci, seules les trois variables Pff, Psf et Nrs présentent des différences significatives entre les individus.

À Gonsé, les quatre variables Ht, Pft, Pff, Lrp et Psr présentent des différences significatives entre les individus.

À Diapangou-Peulh, seules les deux variables Ht et Lrp présentent des différences significatives entre les individus. 
Le tableau III synthétise les résultats de l'analyse de variance inter-populations. Pour les douze variables, on observe des différences significatives à hautement significatives entre les populations, respectivement aux seuils de probabilité P de $5 \%, 1 \%$ et $0,1 \%$.

Les tableaux IV et $\mathrm{V}$ présentent les comparaisons des moyennes des variables des quatre populations respectivement pour les parties souterraine et aérienne des plantules. L'utilisation d'un test de Newman-Keuls permet de classer les populations pour chaque variable.

Pour la partie souterraine, en considérant les différences entre longueurs de la racine principale (Lrp), diamètres de racine principale (Drp) et nombre de racines secondaires (Nrs), on constate que :

- les individus de Diapangou-Peulh investissent dans la croissance linéaire du pivot et dans la densité du système racinaire au détriment de la croissance radiale ;

- à l'inverse, les individus de You investissent dans la croissance radiale et dans la densité du système racinaire, mais moins dans la croissance linéaire du pivot que les trois autres populations ;

- les populations de Yarci et Gonsé occupent des classements intermédiaires entre les deux populations extrêmes pour chacune des trois variables.

Parmi les quatre populations étudiées, les individus de la population de You et de Diapangou-Peulh présentent respectivement et de façon significative la plus forte et la plus faible biomasse racinaire.

Concernant la vigueur de croissance aérienne, on constate que :

- les individus de Gonsé ont la hauteur de tige significativement la plus élevée alors que ceux de Diapangou-Peulh présentent la hauteur de tige significativement la moins élevée des quatre populations, et qui peut être considérée dans l'absolu comme faible ; les individus de You et Yarci occupent des positions intermédiaires ;
Tableau III.

Résultats de l'analyse de variance inter-population.

\begin{tabular}{lrlr} 
Variables & \multicolumn{1}{c}{$\mathbf{F}$} & \multicolumn{1}{c}{$\mathbf{P}$} & ET \\
\hline Ht & 93,15 & $0,0001^{\star \star \star}$ & 8,68 \\
\hline Dc & 61,54 & $0,0001^{\star \star \star}$ & 1,07 \\
\hline Nram & 50,85 & $0,0001^{\star \star \star}$ & 0,22 \\
\hline Pft & 49,06 & $0,0001^{\star \star \star}$ & 2,26 \\
\hline Pst & 41,22 & $0,0001^{\star \star \star}$ & 0,76 \\
\hline Pff & 12,79 & $0,0001^{\star \star \star}$ & 2,02 \\
\hline Psf & 2,57 & $0,006^{\star \star}$ & 1,22 \\
\hline Lrp & 3,02 & $0,03^{\star}$ & 5,01 \\
\hline Drp & 8,87 & $0,0001^{\star \star \star}$ & 2,31 \\
\hline Nrs & 4,15 & $0,009^{\star \star}$ & 6,19 \\
\hline Pfr & 10,08 & $0,0001^{\star \star \star}$ & 10,89 \\
\hline Psr & 10,72 & $0,0001^{\star \star \star}$ & 4,82
\end{tabular}

$P$ : probabilité ; F : F statistique ; ET : écart-type ; $\mathrm{Dc}(\mathrm{mm})$ : diamètre au collet; $\mathrm{Ht}(\mathrm{cm})$ : hauteur de la tige ; Nram : nombre de rameaux; Pft (g) : poids frais de la tige; Pst (g) : poids sec de la tige ; Pff (g) : poids frais des feuilles ; Psf (g) : poids sec des feuilles; $\operatorname{Lrp}(\mathrm{cm})$ : longueur de la racine principale; Drp $(\mathrm{mm})$ : diamètre de la racine principale ; Nrs : nombre de racines secondaires ; Pfr (g) : poids frais de la racine; Psr (g) : poids sec de la racine; $n s$ : non significatif ; ${ }^{*}$ : significatif ; ${ }^{\star \star}$ : très significatif ${ }^{\star \star \star *}$ : hautement significatif.

- l'analyse de la variable Dc (diamètre au collet de la tige) montre que les individus de You ont la plus forte croissance radiale, mais sans différence significative avec Gonsé, tandis que ceux de Diapangou-Peulh ont la croissance radiale significativement la plus faible des quatre populations.

Tableau IV.

Comparaison des moyennes des variables de la partie souterraine des plantules.

\begin{tabular}{l|c|c|c|c|c|c|} 
Populations & \multicolumn{1}{|c|}{$\operatorname{Lrp}(\mathbf{c m})$} & Drp $(\mathbf{m m})$ & Nrs & Pfr (g) & Psr (g) \\
\cline { 1 - 6 } You & $27,55 \mathrm{ab} \pm 6,07$ & $15,23 \mathrm{a} \pm 2,98$ & $22,91 \mathrm{a} \pm 7,40$ & $39,66 \mathrm{a} \pm 14,90$ & $14,19 \mathrm{a} \pm 7,13$ \\
\hline Yarci & $28,12 \mathrm{ab} \pm 6,03$ & $14,64 \mathrm{ab} \pm 3,94$ & $17,93 \mathrm{~b} \pm 6,58$ & $35,62 \mathrm{ab} \pm 15,41$ & $11,38 \mathrm{~b} \pm 5,86$ \\
\hline Gonsé & $28,51 \mathrm{ab} \pm 8,03$ & $13,54 \mathrm{~b} \pm 2,88$ & $16,93 \mathrm{~b} \pm 7,56$ & $30,47 \mathrm{~b} \pm 12,60$ & $9,30 \mathrm{bc} \pm 4,27$ \\
\hline Diapangou- Peulh & $31,7 \mathrm{a} \pm 7,06$ & $12,16 \mathrm{c} \pm 2,19$ & $18,20 \mathrm{~b} \pm 8,79$ & $24,24 \mathrm{c} \pm 11,52$ & $7,10 \mathrm{c} \pm 4,97$
\end{tabular}

Lrp : longueur de la racine principale; Drp : diamètre de la racine principale ; Nrs : nombre de racines secondaires; Pfr : poids frais de la racine; Psr : poids sec de la racine ; \pm écart-type ; les moyennes sur une colonne portant les mêmes lettres ne sont pas significativement différentes au seuil de $5 \%$. 
Tableau V.

Comparaison des moyennes des variables de la partie aérienne des plantules.

\begin{tabular}{|c|c|c|c|c|c|c|c|}
\hline Populations & $\mathrm{Ht}(\mathrm{cm})$ & $\mathrm{Dc}(\mathrm{mm})$ & Nram & Pft (g) & Pst (g) & Pff (g) & Psf (g) \\
\hline You & $39,71 b \pm 6,78$ & $6,75 a \pm 0,97$ & $0,33 c \pm 0,07$ & $7,18 a \pm 2,43$ & $2,11 a \pm 0,89$ & $1,55 c \pm 1,25$ & $0,55 c \pm 0,43$ \\
\hline Yarci & $38,16 b \pm 6,95$ & $6,30 b \pm 1,14$ & $0,63 a \pm 0,02$ & $6,53 a \pm 2,43$ & $1,77 b \pm 0,75$ & $3,00 b \pm 1,64$ & $1,58 a \pm 3,59$ \\
\hline Gonsé & $42,79 a \pm 6,73$ & $6,56 a b \pm 0,97$ & $0,48 b \pm 0,03$ & $7,36 a \pm 2,38$ & $2,30 a \pm 0,94$ & $3,22 b \pm 1,66$ & $1,17 a b \pm 0,5$ \\
\hline $\begin{array}{l}\text { Diapangou- } \\
\text { Peulh }\end{array}$ & $22,97 c \pm 5,31$ & $4,49 c \pm 0,89$ & $0,30 c \pm 0,06$ & $2,87 b \pm 1,23$ & $0,75 c \pm 0,40$ & $4,84 a \pm 2,72$ & $1,15 a b \pm 0,70$ \\
\hline
\end{tabular}

$\mathrm{Ht}$ : hauteur de la tige ; Dc : diamètre au collet ; Nram : nombre de rameaux; Pft : poids frais de la tige ;

Pst : poids sec de la tige ; Pff : poids frais des feuilles; Psf : poids sec des feuilles; \pm écart-type ; les moyennes sur une colonne portant les mêmes lettres ne sont pas significativement différentes au seuil de $5 \%$.

Pour la biomasse aérienne, on constate que :

- pour le poids frais de la tige (Pft), les populations de You, Yarci et Gonsé ne sont pas significativement différentes ; celle de Diapangou-Peulh présente significativement la plus faible biomasse des quatre populations ;

- le poids sec de la tige (Pst) est significativement le plus

Tableau VI.

Héritabilité des caractères selon les variables.

\begin{tabular}{l|c|r|r} 
Variables & $\begin{array}{c}\text { Variance } \\
\text { de l'erreur }\end{array}$ & $\begin{array}{c}\text { Variance } \\
\text { populations }\end{array}$ & $\mathbf{H}^{\mathbf{2}}$ \\
\hline Ht & 76 & 16,76 & 28,31 \\
\hline Dc & 76 & 0,35 & 0,46 \\
\hline Nram & 76 & 0,04 & 0,05 \\
\hline Pft & 76 & 1,80 & 2,43 \\
\hline Pst & 76 & 0,23 & 0,30 \\
\hline Pff & 76 & 2,83 & 3,87 \\
\hline Psf & 76 & 1,41 & 1,89 \\
\hline Lrp & 76 & 23,33 & 44,30 \\
\hline Drp & 76 & 4,10 & 5,71 \\
\hline Nrs & 76 & 34,21 & 81,89 \\
\hline Pfr & 76 & 32,19 & 73,47 \\
\hline Psr & 76 & 17,02 & 28,86
\end{tabular}

$\mathrm{H}^{2}$ : héritabilité au sens large; $\mathrm{Ht}(\mathrm{cm})$ : hauteur de la tige ; Dc ( $\mathrm{mm})$ : diamètre au collet; Nram : nombre de rameaux ; Pft (g) : poids frais de la tige ; Pst (g) : poids sec de la tige ; Pff (g) : poids frais des feuilles; Psf (g) : poids sec des feuilles; Lrp (cm) : longueur de la racine principale; $\operatorname{Drp}(\mathrm{mm})$ : diamètre de la racine principale ; Nrs : nombre de racines secondaires ;

$\operatorname{Pfr}(\mathrm{g})$ : poids frais de la racine ;

Psr (g) : poids sec de la racine. élevé pour les individus de You et Gonsé alors que ceux de Diapangou-Peulh présentent significativement le plus faible poids (résultat logiquement équivalent à celui de la variable précédente) ; les individus de Yarci occupent une position intermédiaire ;

- les individus de Diapangou-Peulh et de You présentent le poids frais de feuilles (Pff) respectivement et significativement le plus élevé et le moins élevé ; les individus de Yarci et Gonsé occupent des positions intermédiaires ;

- les individus de Yarci présentent le poids sec de feuilles (Psf) le plus élevé, mais sans différence significative avec ceux de Gonsé, tandis que ceux de You présentent le poids sec de feuilles significativement le plus faible, résultat identique à celui de la variable précédente Pst ; les populations de Gonsé et Diapangou-Peulh occupent des positions intermédiaires ;

- les individus de Yarci ont le nombre de rameaux (Nram) significativement le plus élevé tandis que ceux de You et Diapangou-Peulh présentent le nombre de rameaux le plus faible, sans différence significative entre les deux populations ; les individus de Gonsé occupent une position intermédiaire.

\section{Héritabilité des caractères}

Le tableau VI présente l'héritabilité des caractères étudiés au stade juvénile. En se basant sur les normes d'héritabilité établies sur les caractères quantitatifs des ligneux forestiers, on distingue quatre groupes de caractères : les six variables Dc, Nram, Pft, Pst, Pff, Psf très peu héritables (héritabilité inférieure à $5 \%$ ) ; la variable Drp peu héritable (héritabilité comprise entre $5 \%$ et $15 \%$ ) ; les deux variables $\mathrm{Ht}$ et Psr qui sont héritables (héritabilité comprise entre $15 \%$ et $30 \%$ ) ; les trois variables Lrp, Nrs et Pfr dont l'héritabilité est supérieure à $30 \%$ et qui sont donc fortement héritables. 
Bois et Forêts des Tropiques - ISSN: L-0006-579X

Volume $344-2^{\text {nd }}$ quarter - July 2020 - p. 33-46

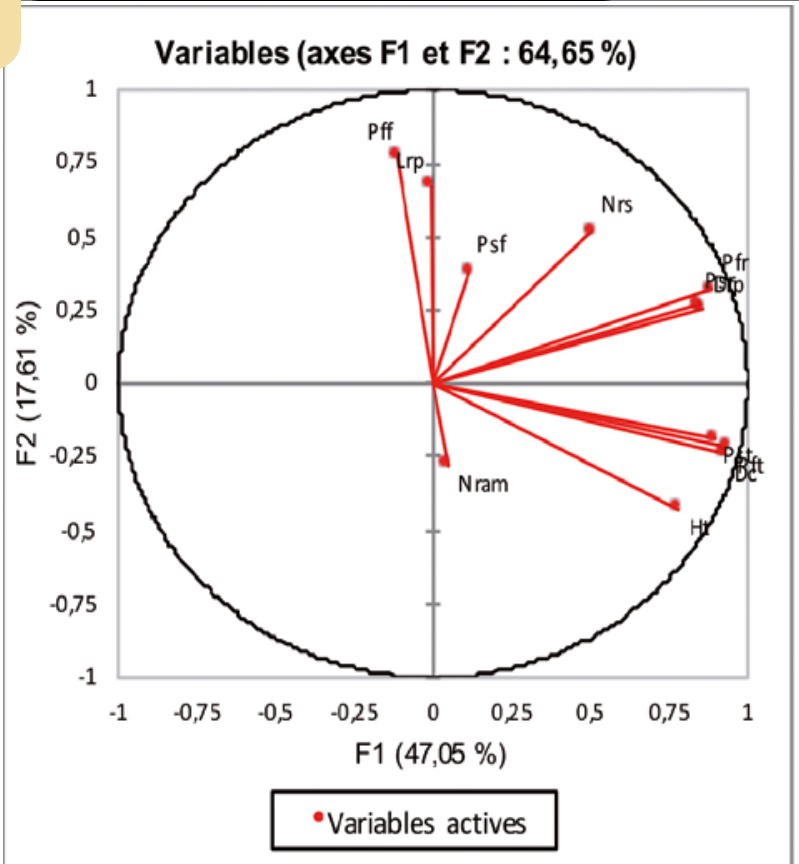

a : ACP des variables dans le plan $1 / 2$

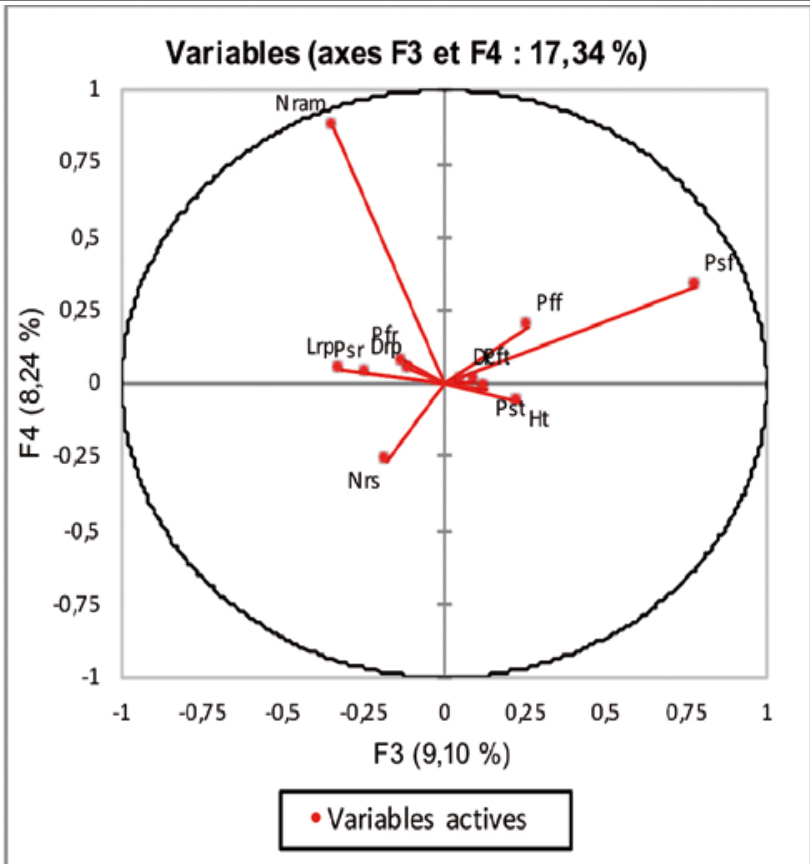

b : ACP des variables dans le plan $3 / 4$

Figure 4.

Corrélation des variables avec les axes F1 et F2 (a) et les axes F3 et F4 (b). Ht (cm) : hauteur de la tige ; Dc (mm) : diamètre au collet ;

Nram : nombre de rameaux; Pft (g) : poids frais de la tige ; Pst (g) : poids sec de la tige ; Pff (g) : poids frais des feuilles ; Psf (g) : poids sec des feuilles ; Lrp $(\mathrm{cm})$ : longueur de la racine principale ; Drp $(\mathrm{mm})$ : diamètre de la racine principale ; Nrs : nombre de racines secondaires;

$\operatorname{Pfr}(\mathrm{g})$ : poids frais de la racine ; Psr (g) : poids sec de la racine.

\section{Descripteurs spécifiques des populations}

L'analyse en composantes principales (ACP) (figure 4) montre que les quatre premiers axes expliquent $81,99 \%$ de la variabilité observée. D'après la figure $4 a$, on constate que les deux premiers axes (F1 et F2) qui expliquent $64,65 \%$ de la variabilité observée concentrent la majorité des variables phénotypiques. D'une part, les variables $\mathrm{Ht}(77 \%)$, Dc (92\%), Pft (93\%), Pst (89\%), Drp (85\%), Nrs (50\%), Pfr (88\%) et Psr (83\%) apparaissent les plus corrélées à l'axe 1 qui peut être défini comme l'axe de la croissance radiale, de la croissance aérienne et de la croissance en biomasse (aérienne et souterraine). D'autre part, les variables Pff (77\%), Lrp (67\%) et Nrs (51\%) sont les plus corrélées à l'axe 2 qui peut donc être considéré comme l'axe de la biomasse fraîche foliaire, de la ramification souterraine et de la croissance linéaire du pivot.

Sur la figure 4b, les axes F3 et F4 qui expliquent 17,34 \% de la variabilité observée ne concentrent que deux variables phénotypiques : la variable Psf (77 \%) est la plus corrélée à

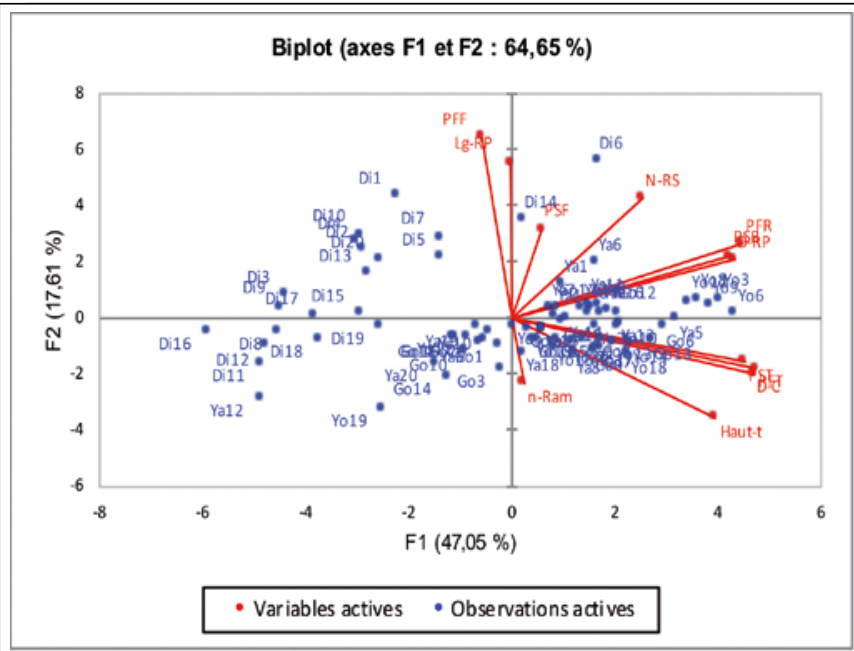

a : représentation des individus et des variables dans le plan $1 / 2$

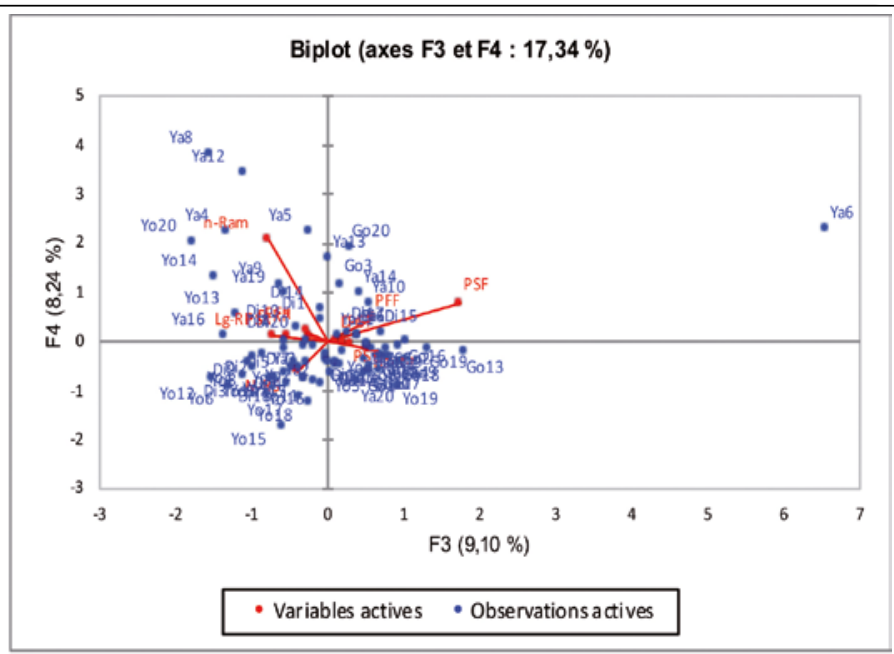

b : représentation des individus et des variables dans le plan $3 / 4$

Yo: You ; Ya : Yarci ; Go : Gonsé; Di : Diapangou-Peulh

Figure 5.

Représentation des individus et des variables dans les plans $1 / 2$ (a) et $3 / 4$ (b). 
Tableau VII.

Rapports entre les variables aériennes et les variables souterraines.

\begin{tabular}{|l|c|c|c|}
\hline Populations & Ht/Lrp & Pfa/Pfs & Psa/Pss \\
\hline You & 1,48 & 0,23 & 0,20 \\
\hline Yarci & 1,37 & 0,27 & 0,31 \\
\hline Gonsé & 1,53 & 0,35 & 0,38 \\
\hline Diapangou-Peulh & 0,75 & 0,32 & 0,29
\end{tabular}
racine principale ; Pfa : poids frais aérien ; Pfs : poids frais souterrain ; Psa : poids sec aérien; Pss : poids sec souterrain.
$\mathrm{Ht}$ : hauteur de la tige; Lrp : longueur de la

de You (15\%) et de Gonsé (10 \%). À l'inverse, aucun individu de Diapangou-Peulh ne présente de ramification. On constate également que le poids sec des feuilles n'est pas un descripteur spécifique à une population donnée.

Le dendrogramme (figure 6) obtenu à partir de la classification ascendante hiérarchisée (CAH), basée sur des distances euclidiennes, fait ressortir trois grands groupes d'individus :

- le groupe 1 (G1) est constitué à 55,88 \% d'individus de Gonsé, 23,52 \% d'individus de Yarci et 20,58 \% d'individus de You ; dans ce groupe on note l'absence d'individus de Diapangou-Peulh ;

- le groupe 2 (G2) est constitué d'un mélange d'individus de You (46,15\%), de Yarci (42,30\%), de Diapangou-Peulh (7,69\%) et de Gonsé (3,84\%) ;

- le groupe 3 (G3) est constitué en quasi-totalité par les individus de Diapangou-Peulh $(90 \%)$ et quelques individus de Yarci (5\%) et de You (5\%).

\section{Compromis d'allocation des ressources nutritives}

l'axe 3 tandis que la variable Nram (87 \%) est la plus corrélée à l'axe 4.

En considérant le biplot $1 / 2$ (figure $5 \mathrm{a}$ ) qui superpose le plan $1 / 2$ des individus au plan $1 / 2$ des variables, on observe le positionnement des individus en fonction des variables qui leur sont spécifiques. Ainsi, on note que la majorité des individus de Diapangou-Peulh se caractérise principalement par une forte croissance de la racine principale et une biomasse foliaire fraîche élevée. Ils se caractérisent également par une faible croissance en hauteur et en diamètre de la tige ainsi qu'une faible biomasse de la tige et des racines. À l'inverse, la majorité des individus de You, de Yarci et de Gonsé présente une racine principale plus courte avec une biomasse foliaire beaucoup plus faible. Ces populations se caractérisent également par une forte croissance en hauteur et en diamètre de la tige ainsi qu'une biomasse élevée de la tige et des racines.

Dans le biplot $3 / 4$ (figure 5 b) qui superpose le plan $3 / 4$ des individus au plan $3 / 4$ des variables, on constate que la ramification est une caractéristique des individus de Yarci (50\%), suivants :

- hauteur de la tige $(\mathrm{Ht})$ / longueur de la racine principale (Lrp) ;

- poids frais aérien (Pfa) / poids frais souterrain (Pfs) ;

- poids sec aérien (Psa) / poids sec souterrain (Pss).

On note dans les populations de You, Yarci et Gonsé une croissance en hauteur de la tige supérieure à la croissance de la racine principale, contrairement à la population de Diapangou-Peulh.

Cependant, le rapport entre la biomasse aérienne (fraîche et sèche) et la biomasse souterraine (fraîche et sèche) est systématiquement inférieur à 1 quelle que soit la population considérée.
Dans le tableau VII sont présentés les trois rapports

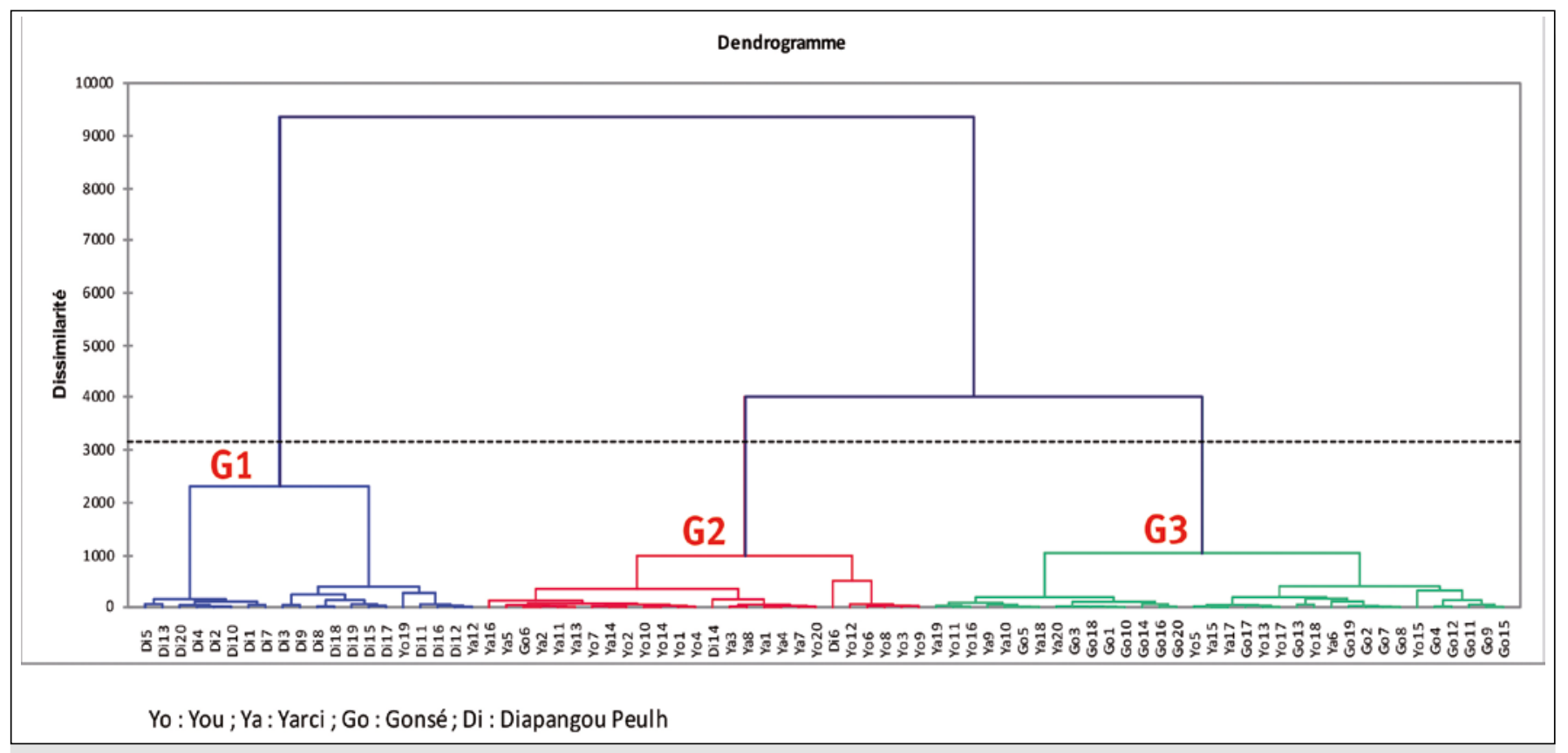

Figure 6.

Regroupement des individus en fonction des caractères phénotypiques. 


\section{Discussion}

Les résultats relatifs à la stratégie de levée indiquent que celle-ci est de type groupé quelle que soit la population considérée. Il ne s'agit donc pas d'une stratégie adaptative spécifique à telle ou telle population mais d'une caractéristique propre à l'espèce. De ce fait, la première sous-hypothèse n'est pas vérifiée. Ce type de levée groupée pourrait être une stratégie évolutive dont la fonction adaptative serait de saturer les besoins des herbivores afin que certaines plantules échappent à l'herbivorie. En effet, Poissonnet (2002) note que les jeunes plants de Sclerocarya birrea en régénération naturelle sont victimes de broutage principalement par les petits ruminants et les rongeurs dès les deux premières semaines de levée. Ceux-ci cessent de l'être dès le début de la lignification de la plantule. Dans les zones tropicales sèches, l'herbivorie constitue un facteur négatif pour la régénération naturelle par graine, compromettant ainsi la bonne dynamique des peuplements. De ce fait, cette stratégie de levée groupée qui assure la survie de quelques plantules à chaque cycle de reproduction permettrait de maintenir des populations à générations chevauchantes chez le prunier d'Afrique.

L'analyse de variance montre une grande variabilité intra-population des caractères clés d'adaptation tels que la hauteur de la tige, la longueur de la racine, la ramification du système racinaire, la biomasse aérienne et la biomasse souterraine.

En référence aux travaux de Hamrick et Godt (1996), selon lesquels le niveau de diversité des populations d'une espèce est le premier paramètre qui conditionne leur survie dans les environnements changeants, on peut considérer que les quatre populations de Sclerocarya birrea étudiées ne sont pas menacées sur le court terme. Cependant, Diallo et al. (2007) mentionnent qu'une telle situation peut rapidement changer en relation avec les actions anthropiques et en l'absence d'importants flux de gènes. En se référant aux travaux de Hall (2005), on peut considérer que cette diversité intra-population pourrait être le résultat de la dissémination des graines par la faune ongulée, les ruminants domestiques, les chauves-souris et les primates. Ainsi, une forme de mutualisme entre l'espèce et ces disperseurs de graines pourrait s'être établie au cours de l'évolution. En effet, ces disperseurs de graines profitent des fruits en tant qu'aliment et l'espèce bénéficie de la dispersion des graines sur de longues distances. Selon Tybirk (1991), ce mode de dissémination contribue au maintien de la diversité génétique à l'intérieur des populations des ligneux forestiers. À ce sujet, Diallo et al. (2014) ont signalé que l'absence d'une dispersion sur de longues distances entraînerait à terme l'apparition d'individus consanguins dans le cas des espèces barochores comme le prunier d'Afrique.

L'analyse de variance, qui fait ressortir des différences significatives inter-populations pour la quasi-totalité des variables mesurées, et la comparaison des moyennes mettent en évidence la grande diversité phénotypique de Sclerocarya birrea dès le stade pépinière. Cette diversité inter-populations observée confirme la deuxième sous-hypothèse : pour un caractère phénotypique donné, certaines populations se différencient des valeurs moyennes de l'espèce pour les quatre populations étudiées. Cette différenciation phénotypique en fonction des zones écologiques vient confirmer la différenciation génétique mise en évidence in situ par Kando et al. (2012) sur les populations de Sclerocarya birrea au Burkina Faso.

La comparaison variable par variable des moyennes des populations permet d'établir différents classements des populations. Ce qui traduit l'expression différentielle des caractères en fonction des zones écologiques. De telles observations avaient été notées par Assogbadjo et al. (2006) pour les populations in situ de Adansonia digitata L. De ce fait, lorsqu'il s'agira de prendre en compte le système racinaire lors des plantations sur différents types de sols, on pourra affecter le matériel végétal originaire de Diapangou-Peulh sur des sols à nappe phréatique profonde alors que celui de You devra être affecté préférentiellement aux sols filtrants. De telles observations ont été mentionnées par Sandwidi et al. (2019) sur les ethno-variétés de Vitellaria paradoxa C.F. Gaertn. Ces résultats permettent de définir un indicateur dans le choix des sites de plantation en fonction des caractéristiques du matériel végétal.

Les calculs d'héritabilité qui conduisent à définir quatre niveaux d'héritabilité pour les 12 variables confirment qu'au jeune âge certains caractères sont peu héritables alors que d'autres le sont fortement. De ce fait, la troisième soushypothèse est vérifiée. Partant de la classification de Nanson (2004) sur l'héritabilité des caractères morpho-adaptatifs des ligneux forestiers, il ressort de l'étude qu'au jeune âge la biomasse aérienne et la croissance radiale sont peu héritables tandis que la croissance en hauteur de la tige, la longueur de la racine principale, le nombre de racines secondaires et la biomasse racinaire sont fortement héritables. Ceci va à l'encontre des résultats de Weber et al. (2015) sur Balanites aegyptiaca qui montre que les paramètres de croissance ne sont pas héritables au jeune âge. Toutefois, si on se réfère aux travaux sur Tamarindus indica conduits par Diallo (sous presse), il est possible que, chez Sclerocarya birrea, ces héritabilités se modifient quand les plants seront en milieu réel, où ils seront soumis à d'autres forces de sélection telles que la sélection naturelle et les mutations.

Les biplots de l'analyse en composantes principales $(A C P)$ révèlent que chaque population présente des caractères phénotypiques qui lui sont spécifiques. Ce résultat valide la quatrième sous-hypothèse et confirme les travaux de Legay et Debouzie (1985), qui montrent que les caractères phénotypiques de chaque population ont été modelés au cours de l'évolution pour répondre aux exigences de son environnement. De tels résultats ont également été obtenus par Chevalier et al. (2003) sur Balanites aegyptiaca, Diallo et al. (2010) sur Tamarindus indica, et Sandwidi et al. (2019) sur Vitellaria paradoxa.

Le dendrogramme de la classification ascendante hiérarchisée (CAH) met en évidence trois groupes distincts. L'observation de ce dendrogramme conduit à constater que, lorsque les individus de Gonsé sont présents en forte proportion, ceux de Diapangou-Peulh sont quasi absents et réciproquement. Ceci permet de considérer que les individus de Gonsé et ceux de Diapangou-Peulh sont phénotypiquement éloignés. À l'inverse, les individus de You et de Yarci constituent un groupe homogène ; ces deux populations sont phénotypiquement proches. On observe également une distribution de quelques individus de You et Yarci dans les deux groupes dominés respectivement par les individus de Gonsé et de Diapan- 
gou-Peulh. Cette constatation pourrait conduire à considérer l'existence d'une métapopulation en cours de fragmentation dont l'isolement génétique est encore inachevé du fait d'un important flux de graines assuré par les chauves-souris et les ruminants domestiques en transhumance.

Le rapport de la biomasse aérienne sur la biomasse souterraine est inférieur à 1 quelle que soit la population. Ce résultat révèle une allocation préférentielle des ressources nutritives au profit du développement du système racinaire. Toutefois, on note qu'au jeune âge cette caractéristique est indépendante de l'aire d'origine des semences. De ce fait, la cinquième sous-hypothèse n'est pas vérifiée. En effet, selon Billand et Diallo (1991), Vandenbelt (1991) et Weber et al. (2018), de nombreuses espèces ligneuses adaptées aux milieux semi-arides investissent une grande partie de leur réserve en hydrates de carbone dans le système racinaire. Cette stratégie dans l'allocation des ressources nutritives permet aux racines des plantes d'atteindre rapidement la nappe phréatique et/ou d'explorer les horizons superficiels du sol en cas de faible pluie, grâce à un système racinaire secondaire et tertiaire abondant (Bassolé, 2018).

\section{Conclusion}

Au terme de cette étude, il ressort que la stratégie de levée groupée qui assure la dynamique des populations basée sur des générations chevauchantes est une donnée essentielle dans les prises de décisions des plans d'aménagement des forêts tropicales sèches. La forte variabilité des caractères phénotypiques intra- et inter-populations ainsi que l'existence des caractères spécifiques à chaque population s'opposent à l'impasse évolutive. Ces résultats augurent d'importants gains génétiques pour la sélection des porte-greffes destinés à la création de variétés clonales. On peut noter également que cette diversité intra- et inter-populations permet d'envisager la sélection individuelle et populationnelle. De ce fait, pour un caractère recherché tel que la longueur de la racine (Lrp) et/ ou le nombre de racines secondaires (Nrs), dans un contexte d'adaptation, la sélection portera sur les meilleurs individus dans les meilleures populations. Il ressort également de cette étude que la forte héritabilité des caractères clés d'adaptation pourrait servir de base de sélection lors de l'affectation du matériel végétal sur les sites de plantation. Le compromis d'allocation des ressources au profit de la croissance du système racinaire montre que Sclerocarya birrea peut avoir une faculté d'adaptation sur des sols filtrants à nappe phréatique profonde ou des sols à glacis à nappe phréatique profonde ou peu profonde. Compte tenu de ces résultats, il apparaît que, dès le jeune âge, il est possible d'opérer une sélection précoce sur la faculté d'adaptation de Sclerocarya birrea à diverses conditions écologiques.

\section{Remerciements}

Ce travail a été réalisé grâce au soutien financier du Fonds national de la recherche et de l'innovation pour le développement (FONRID). À travers cet article, nous lui exprimons notre reconnaissance pour l'intérêt porté à la valorisation des fruitiers locaux au Sahel.

\section{Références bibliographiques}

Assogbadjo A. E., Kyndt T., Sinsin V., Gheysen G., Van Damme P., 2006. Patterns of genetic and morphometric diversity in baobab (Adansonia digitata) populations across different climatic zones of Benin (West Africa). Annales de Botanique, 97 (5): 819-830. https://doi.org/10.1093/aob/mcl043

Avana T. M. L. A., Kéouna S., Dongock N. D., Mouga M. B., 2019. Structure des peuplements et potentiel de domestication de Parkia biglobosa dans la région de Tandjilé-Ouest (Tchad). International Journal of Biological and Chemical Science, 13 (1) : 219-236. https://doi.org/10.4314/ijbcs.v13i1.19

Bassolé D. M. S., 2018. Étude comparée des caractéristiques juvéniles de cinq ethno-variétés de karité Vitellaria paradoxa C. F. Gaertn Hepper (Sapotaceae) au Burkina Faso. Mémoire d’ingénieur du développement rural, option eaux et forêts, Université Nazi Boni, Burkina Faso, 49 p.

Billand A., Diallo B. O., 1991. Amélioration des ligneux soudanosahéliens : Stratégies et perspectives. Rapport d'activité 19901991. Ouagadougou, Burkina Faso, MESSRS/CNRST/INERA/DPF, $196 \mathrm{p}$.

Chevalier M. H., Bensaid S., Diallo O. B., Shaki R., Ganaba S., Sanou J., et al., 2003. Biodiversité et multidisciplinarité : méthodologie pour les zones arides. Bois et Forêts des Tropiques, 276 (2) : 33-41. https://revues.cirad.fr/index.php/BFT/article/view/20195

Dagnelie P., 1998. Statistique théorique et appliquée. Bruxelles, Belgique, De Boeck \& Larcier, 517 p.

Diallo B. 0., 2017. Cours de génétique appliquée aux arbres forestiers en zone soudano-sahélienne. Ouagadougou, Burkina Faso, $32 \mathrm{p}$.

Diallo B. O., Bastide B., Poissonnet M., Dao M., Sanou J., Hossaert-Mc Key M., 2006. Mise en évidence d'une androdioécie morphologique et d'une " hétérostigmatie » chez Sclerocarya birrea (A. Rich.) Hochst. Fruits, 61 (4) : 259-266. https://doi.org/10.1051/ fruits:2006023

Diallo B. O., Dao M. E. C., Sanou J., Sanon K., Dianda M., Cao V., 2012. Essai de provenances de Acacia senegal (L.) Willd. en zone sahélienne du Burkina Faso : Adaptation et production de gomme. Science et Technique, Sciences Naturelles et Agronomie, 32 (2): 47-63.

Diallo B. O., Joly H. I., Hossaert-Mc Key M., McKey D., Chevallier M. H., 2007. Genetic diversity of Tamarindus indica populations: Any clues on the origin from its current distribution? African Journal of Biotechnology, 6 (7): 853-860. https://doi.org/10.5897/ AJB2007.000-2098

Diallo B. O., Joly H. I., Hossaert-Mc Key M., McKey D., Chevallier M. H., 2010. Variation des caractères biométriques de neuf provenances de Tamarindus indica L. (Caesalpinioideae). Fruits, 65 (2) : 153167. https://doi.org/10.1051/fruits/2010010

Diallo B. O., Ouédraogo M., Chevallier M. H., Joly H. I., Hossaert-Mc Key M., McKey D., 2014. Potential pollinators of Tamarindus indica L. (Caesalpinioideae) in Sudanian region of Burkina Faso. African Journal of Plant Science, 8 (12): 528-536.

Diallo B. O., Sanou J., Dao M. E. C., Cao V., Assimi S., 2000. Amélioration génétique des ligneux soudano-sahéliens. Rapport final d'activité du projet FAC/94/CD/78/BKA, CNRST/INERA/DPF, Ouagadougou, Burkina Faso, 199 p.

Direction générale des forêts (DGF), 2016. Monographie nationale sur la diversité biologique du Burkina Faso. Ouagadougou, Burkina Faso, $170 \mathrm{p}$. 
Faye M. D., Weber J. C., Abasse T. A., Boureima M., Larwanou M., Bationo B. A., et al., 2011. Farmers preferences for tree functions and species in West African Sahel. Forests, Trees and Livelihoods, 20: 113-136. https://doi.org/10.1080/14728028.2011.9756702

Fontès J., Guinko S., 1995. Carte de la végétation et de l'occupation des sols du Burkina Faso. Note explicative. Toulouse, France, ministère de la Coopération française, Projet Campus, 67 p.

Garnier C., 1972. Stockholm conférence 1972. Encyclopædia universalis. https://www.universalis.fr/encyclopedie/conferencede-stockholm/

Gutman F., Nerd A., Mizrahi Y., Bar-Zvi D., Raveh D., 1999. Application of random amplified polymorphic DNA markers for identification of marula genotypes. Horticultural Science, 34: 1256-1258. https:// doi.org/10.21273/hortsci.34.7.1256

Hall J. B., 2002. Ressources végétales de l'Afrique tropicale. In : Précurseur-Programme PROTA Wageningen, Pays-Bas, 144-148.

Hall J. B., 2005. Sclerocarya birrea (A. Rich.) Hocsht. Anacardiaceae. In: Janick J., Paull R. (eds). Encyclopedia of fruits and nuts. Wallingford, UK, CAB International.

Hamrick J. L., Godt M. J. W., 1996. Conservation genetics of endemic plant species. In: Avise J. C., Hamrick J. L. (eds). Conservation genetics: case histories from nature. New York, USA, Chapman and Hall, 281-334.

Hébert Y., Vincourt P., 1985. Mesures de la divergence génétique. Distances calculées sur les critères biométriques. In : Lefort-Buson M., de Vienne D. (éds). Les distances génétiques : estimation et applications. Paris, France, Institut national de la recherche agronomique, $181 \mathrm{p}$

Kando B. P., Bisseye C., Nanéma K. R., Traoré R. E., Yé H., Diallo O. B., et al., 2012. Genetic diversity of Sclerocarya birrea subspecies birrea populations in Burkina Faso detected by RAPDs. African Journal of Biotechnology, 11 (1): 99 -108. https://doi.org/10.5897/ AJB11.980

Kando B. P., Zongo J. D., Nanema R. K., Traoré E. R., 2008. Étude de la variation de quelques caractères morphologiques d'un échantillon de Sclerocarya birrea au Burkina Faso. International Journal of Biological and Chemical Science, 2 (4): 549-562. https://doi. org/10.4314/ijbcs.v2i4.39765

Lamien N., Bayala J., 1996. Rôle social et économique de l'arbre dans le milieu rural. Aspects utilisation et commercialisation de quelques produits non ligneux dans l'Ouest du Burkina. Rapport technique : analyse de la campagne agricole 1995-1996. Ouagadougou, Burkina Faso, INERA, 28 p

Legay J. M., Debouzie F. A., 1985. Introduction à la biologie des populations. Paris, France, Masson, $512 \mathrm{p}$.

Muok B. O., Khumalo S. G., Tadesse W., Alem S., 2011. Conservation et utilisation durable des ressources génétiques des espèces ligneuses alimentaires prioritaires de l'Afrique subsaharienne : Sclerocarya birrea, prunier d'Afrique. Biodiversity International (Saforgen), INIA, CITA, 12 p. http://hdl.handle.net/10532/1690

Muok B. O., Matsumura A., Ishii T., Odee D. W., 2007. Genetic diversity of Sclerocarya birrea populations in Kenya. Journal of Arid Environments, 71: 1-11. https://doi.org/10.1016/i. jaridenv.2006.11.007

Nanson A., 2004. Génétique et amélioration des arbres forestiers. Gembloux, Belgique, Presses agronomiques de Gembloux, 712 p.

Poissonnet M., 2002. Potentialités de régénération par graines de Sclerocarya birrea (A. Rich.) Hochst. dans une forêt tropicale sèche aménagée. Mémoire DESS, Université Paris XII, France, Ouagadougou, Burkina Faso, 70 p.

Sandwidi A., Bassolé D. M. S., Diallo B. O., Sawadogo M., 2019. Variabilité des caractères morphologiques des plantules de cinq ethno-variétés de karité (Vitellaria paradoxa C.F. Gaertn, Sapotaceae) au Burkina Faso. International Journal of Biological and Chemical Science, 13 (2) : 811-823. https://doi.org/10.4314/iibcs.v13i2.19
Shackleton C., 2002. Growth and fruit production of Sclerocarya birrea in the South African lowveld. Agroforestry Systems, 55: 175180. https://doi.org/10.1023/A:1020579213024

Tybirk K., 1991. Régénération des légumineuses ligneuses du Sahel. Aarhus, Danemark, Botanical Institute Aarhus University, 86 p.

Vandenbelt R. J., 1991. Rooting systems of western and southern African provenances of Faidherbia albida (Del.) A. Chev. (Syn. Acacia albida Del.) - a comparative analysis with biogeographic implications. Agroforestry Systems, 14: 233-244. https://doi. org/10.1007/bf00115738

Weber J. C., Sotelo M. C., Kalinganire A., Abasse T., Larwanou M., 2015. Genetic variation and clines in growth and survival of Prosopis africana from Burkina Faso and Niger: comparing results and conclusions from a nursery test and a long-term field test in Niger. Euphytica, 205: 809-821. https://doi.org/10.1007/s10681015-1413-4

Weber J. C., Sotelo M. C., Soumana I., Diallo B. O., Abasse T., Larwanou M., Bationo B. A., 2018. Genetic and geographic variation in growth of Balanites aegyptiaca in Niger: comparing results from provenance/progeny tests in the nursery and field. New Forests, 50 (4): 13-32. https://doi.org/10.1007/s11056-018-9686-9

\section{Diallo et al. - Contribution des auteurs}

\section{RÔLE DU CONTRIBUTEUR NOMS DES AUTEURS}

\begin{tabular}{|c|c|}
\hline Conceptualisation & F. A. Diallo, B. O. Diallo \\
\hline Gestion des données & F. A. Diallo \\
\hline Analyse formelle & A. Sandwidi \\
\hline $\begin{array}{l}\text { Acquisition } \\
\text { du financement }\end{array}$ & B. O. Diallo \\
\hline Enquête et investigation & F. A. Diallo, A. Sandwidi \\
\hline Méthodologie & $\begin{array}{l}\text { F. A. Diallo, B. O. Diallo, } \\
\text { M. E. C. Dao, } \\
\text { P. Bationo-Kando }\end{array}$ \\
\hline Gestion de projet & B. O. Diallo \\
\hline Ressources & $\begin{array}{l}\text { Institut de l'environnement } \\
\text { et de recherches agricoles } \\
\text { (INERA/Burkina Faso) }\end{array}$ \\
\hline Logiciels & A. Sandwidi \\
\hline Supervision & $\begin{array}{l}\text { P. Bationo-Kando, } \\
\text { M.E.C. Dao, B.O. Diallo }\end{array}$ \\
\hline Validation & $\begin{array}{l}\text { P. Bationo-Kando, } \\
\text { M. E. C Dao, B. O. Diallo }\end{array}$ \\
\hline Visualisation & F. A. Diallo, A. Sandwidi \\
\hline $\begin{array}{l}\text { Écriture - Préparation } \\
\text { de l'ébauche originale }\end{array}$ & F. A. Diallo \\
\hline
\end{tabular}

Bois et Forêts des Tropiques - Revue scientifique du Cirad
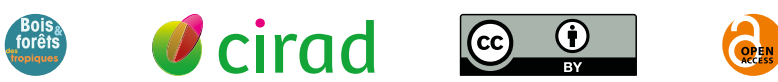

Cirad - Campus international de Baillarguet, 34398 Montpellier Cedex 5, France - Contact : bft@cirad.fr - ISSN : L-0006-579X 